(arsenic and copper), solvents (toluene and xylene), flame retardants, and ethylene glycol ethers.

Conclusions Some endocrine disruptors may play a role in the aetiology of certain lymphoma subtypes. Limitations in interpreting our findings include time- and country-related changes in exposure not reflected by the JEM, multiple comparisons and nondifferential misclassification leading to the attenuation of estimates for binary exposures.

Additional authors: Nikolaus Becker, Paolo Boffetta, Paul Brennan, Lenka Foretova, Marc Maynadie, Anthony Staines, Alexandra Nieters

\section{OCCUPATIONAL AND GENETIC RISK FACTORS FOR MYELOPROLIFERATIVE NEOPLASMS (MPN): A CASE- CONTROL STUDY}

CG Gross-Davis, Burstyn, Heavner, Klotz, Lynch, Newschaffer, Santella, Frank. Drexel Univeristy, Philadelphia, United States of America

\subsection{6/oemed-2013-101717.382}

Objectives The aetiology of a rare category of myeloproliferative neoplasms (MPN), bone marrow diseases with an excess of blood cells, is currently unknown. An MPN cluster in northeastern Pennsylvania allowed investigation of occupational risk factors and gene-environment interactions. Among our hypotheses were risks associated with aromatic and heterocyclic amines.

Methods This 2011 population-based case-control study assessed lifetime occupational, residential, smoking and dietary history by telephone interview. Cases $(n=55)$ were identified from the Pennsylvania cancer registry and a previous MPN study. Controls ( $\mathrm{n}=$ 473) were selected based on eligibility screening using random digit dialling. People born from 1921-1968 and residing in 3 counties with high incidence of MPN were eligible. Blood samples for genotyping were collected from 31 cases and 292 controls.

Results Cases were older (median age $=71$ vs $61 \mathrm{yrs}$ ) and more likely to be male (49\% vs 39\%) compared to controls but otherwise demographically similar. Ever working in ten employment areas (welding, painting, degreasing, firefighting or working with glue, solvents/inks, pesticides, diesel equipment, animals, or Xrays/radioactive material at the 8 most recent jobs) were not associated with MPN.

In analyses that examined the main effects of over 50 environmentally sensitive genes, the presence of NAT2 slow acetylator genotype, GSTM1 gene deletion, and GSTA1, and GSTM3 variants were associated with an increased risk for MPNs (unadjusted ORs 2.1-3.2, 95\% C. I.s excluding 1.0). Results were similar for analyses restricted to JAK2 positive cases.

Conclusions No relationship was found with occupations with presumed exposure to aromatic and heterocyclic amines, but our findings suggest that genotypes that modify the toxicity of these exposures may play a role in MPNs. Sources of exposures important to the pathway whereby NAT2 or other genotypes modify the effect of exposures in this population remain unclear and there is ongoing work on refining exposure assessment in the project.

\section{Session: 31. Exposure assessment IV}
383 DIFFERENT APPROACHES TO ESTIMATE EXPOSURE TO WORK STRESSORS, USING REPEATED MEASUREMENTS, AND THE ASSOCIATION WITH CARDIOVASCULAR DISEASE

\section{K Szerencsi, van Amelsvoort, Prins, Kant. Maastricht University, Maastricht, Nederland}

\subsection{6/oemed-2013-101717.383}

Objectives To explore the stability of exposure to work stressors over time and to examine the impact of different approaches of estimating exposure on the cardiovascular disease (CVD) risk estimation.

Methods The Maastricht Cohort Study-Work Stressor Score (MCS-WSS) was used to assess work stressors at three consecutive time points among 6154 employees participating in the MCS. Incident CVD was assessed with questionnaires. Five approaches were used to estimate exposure as: e.g. single exposure assessment, cumulative exposure above a cutoff point, total exposure and average exposure. The association between work stressors and CVD was stepwise adjusted for age, gender, educational level, smoking, body mass index, hypertension, leisure physical activity and negative affectivity.

Results The correlation between the work stressor scores assessed at the first and third time point was 0.58. Employees with a stable exposure above the highest quartile had a fully adjusted HR of 1.58 (95\% CI: 0.93-2.72). Employees with the highest quartile total exposure had a fully adjusted HR of 1.22 (95\% CI: 0.77-1.95) as compared to employees with the lowest quartile dose. Employees in the upper quartile of the average MCS-WSS had a fully adjusted HR of 1.26 (95\% CI: 0.79-2.01) as compared to employees in the lowest quartile of the MCSWSS. Employees with a single exposure assessment had a fully adjusted HR of 0.91 (95\% CI: 0.62-1.33).

Conclusions Employees with a stable exposure above the highest quartile score during a minimum of two years have the highest relative CVD risk.

\section{\begin{tabular}{|l|l}
384 & SKIN PHYSIOLOGY-BASED EVENTS IN
\end{tabular} THERMOREGULATION FOLLOWING EXPOSURE TO SIMULATED SOLAR RADIANT HEAT}

C P Chen, Chang, Chen. China Medical University, Taichung, Taiwan

\subsection{6/oemed-2013-101717.384}

Objectives The exposure to solar radiant heat (SRH) presents a risk of thermal stress. The risk is exacerbated when people have to experience an instantaneous change in the thermal environment in form of a temperature step due to loss of SRH, e.g., when walking into a thermal transient near a building entrance. This study investigated the skin physiology-based thermoregulation in response to SRH exposure and that occurred when experiencing a temperature step.

Methods The study was conducted in twin climate-controlled chambers, with the first chamber simulating a thermal exposure involving SRH (the outdoor chamber) and the second an environment maintained at a constant $24^{\circ} \mathrm{C}$ without SRH exposure (the indoor chamber). The temperature in the outdoor chamber was $28^{\circ} \mathrm{C}$, with the $\mathrm{SRH}$ present being equivalent to an increase in globe temperature of $4^{\circ} \mathrm{C}$. Ten male and female participants each first sat in the outdoor chamber for $30 \mathrm{~min}$ and were monitored for change in skin capillary blood flow (SCBF), skin moisture, and transepidermal water loss (TEWL), and then moved swiftly into the indoor chamber and were monitored for another $30 \mathrm{~min}$.

Results Following SRH exposure the SCBF increased in females but not in males. When experiencing the temperature drop, the $\mathrm{SCBF}$ required a longer period to decrease than the reduction 
observed when the drop was attributed entirely to loss of convective heat. For males, the most pronounced change in thermoregulation was in skin moisture and TEWL, suggesting a greater thermal load from SRH exposure in males that required water evaporation from the skin to regain thermal balance.

Conclusions The exposure to SRH presented unique influences to core metabolism and thermoregulation compared to those from exposure to convective heat. Adequate clothing protecting against temperature step and the residual influence of SRH was necessary in thermal transient.

\section{CALIFORNIA HEAT ILLNESS PREVENTION STUDY (CHIPS) IN IMMIGRANT LATINO FARM WORKERS}

'B Schenker, 'Mitchell, 'Wadsworth, 'Jones. 'University California, Davis, Davis, United States of America; ${ }^{2}$ California Institute for Rural Studies, Davis, California, United States of America

\subsection{6/oemed-2013-101717.385}

Objectives To quantify the contributions of work intensity and ambient heat to the risk of heat illness in field labourers working with diverse crops.

Methods Field workers were assessed throughout a single daytime work shift. Body weight and blood osmolality changes, continuous heart rate and core body temperature (using ingested telemetry) were recorded. Personal and stationary (area) ambient air temperature and relative humidity were measured using individual data loggers and weather stations. Workers were questioned about their current and historical experiences with field work in the heat.

Results One-hundred workers were assessed between late June and August 2012 on seven farms in the California Central Valley. Thirteen were female, mean age was $36.8(\mathrm{SD}=11.9)$ years. Ninety-four percent were born in Mexico, the remainder in the USA, and over $95 \%$ of the workers identified as Latino. Educational level was low; $47 \%$ had attended $\leq 6$ years of school. Twenty-two percent lost over $1.5 \%$ of their original body weight (ACGIH suggested criteria for increased risk of dehydration), $80.2 \%$ increased serum osmolality, with $21 \%$ increasing $\geq 3 \%$. Males lost significant weight $(-0.56 \mathrm{~kg}, 95 \% \mathrm{CI}-0.40$ to -0.73 $\mathrm{kg})$ as did those whose blood osmolality increased $(-0.59 \mathrm{~kg}$, 95\% CI -0.34 to $-0.85 \mathrm{~kg}$ ). Associations will be assessed between heart rate, task, crop, ambient conditions and core body temperature.

Conclusions Field workers exhibited reduced hydration status over their work shift. Combined physiological and survey data may allow susceptibility to heat illness in summertime agricultural workers to be quantified.

\section{ANNOYANCE FROM OCCUPATIONAL NOISE: THE IMPACT} OF EXPOSURE LEVEL AND HEARING PROTECTION

${ }^{1}$ A Kolstad, 'Stokholm, 'Vestergaard, ${ }^{1}$ Frederiksen, ${ }^{2}$ Hansen, ${ }^{3}$ Lund, ${ }^{3}$ Kristiansen, ${ }^{1}$ Christensen, ${ }^{4}$ Wetke, ${ }^{5}$ Bonde. 'Aarhus University Hospital, Aarhus C, Denmark; ${ }^{2}$ Copenhagen University, Copenhagen, Denmark; ${ }^{3}$ The National Research Centre for the Working Environment, Copenhagen, Denmark; ${ }^{4}$ Odense University Hospital, Odense, Denmark; ${ }^{5}$ Bispebjerg Hospital, Copenhagen, Denmark

\subsection{6/oemed-2013-101717.386}

Objectives. Annoyance from transportation noise is well characterised but little is known about occupational noise. We investigated the relation between occupational noise exposure level, the use of hearing protection devices (HPD), and noise annoyance.
Methods We studied 452 industrial workers and 68 financial workers who post shift reported noise annoyance during work on a 5 -point scale. Noise exposure level was recorded every 5 seconds at the dominant shoulder for 24 hours and we calculated the $\mathrm{L}_{\mathrm{Aeq}}$ value for work hours. For 342 workers who kept a HPD diary, we subtracted $10 \mathrm{~dB}$ from every noise recording obtained during HPD use and estimated the $\mathrm{L}_{\mathrm{AEq}}$ value at the ear.

Results The mean measured noise exposure level was $80.0 \mathrm{~dB}$ (A) [range: 55.0-98.9] and the mean estimated level at the ear $77.8 \mathrm{~dB}(\mathrm{~A})$ [range: 55.0-94.2]. Fifty-one percent of workers exposed at a measured noise level $\geq 85 \mathrm{~dB}(\mathrm{~A})$ were annoyed (the upper 3 points on the annoyance scale) and 14\% highly annoyed (the upper 2 points). In a logistic regression model that also adjusted for neuroticism, annoyance increased monotonously with $6 \%$ per $\mathrm{dB}(\mathrm{A})(\mathrm{OR}=1.06,95 \%$ CI 1.02-1.09). A slightly lower trend was seen per estimated $\mathrm{L}_{\mathrm{AEq}}$ level at the ear $(\mathrm{OR}=1.04,95 \%$ CI $0.99-1.07)$. HPD use was strongly associated with annoyance when adjusted for noise exposure level (OR 2.3, 95\% CI 1.3-3.9).

Conclusions Increasing occupational noise exposure level was associated with increasing prevalence of annoyance but at a much lower annoyance level than seen for transportation noise. We documented no gainful effect of hearing protection, but the opposite. An obvious explanation is that noise annoyance is not solely a question of the amount of noise appearing at the ear but also other characteristics of a noisy work environment; furthermore, that HPD use is predicted by noise sensitivity and other individual characteristics that are associated with noise annoyance.

\section{EXPOSURE TO PARTICLES AND NOISE DURING HIGHWAY MAINTENANCE WORK AND ASSOCIATED SHORT-TERM CARDIOVASCULAR HEALTH EFFECTS}

${ }^{1} \mathrm{R}$ M Meier, ${ }^{2}$ Cascio, ${ }^{2}$ Ghio, ${ }^{1}$ Danuser, ${ }^{1}$ Riediker. ${ }^{1}$ Institute for Work and Health, University of Lausanne, Lausanne, Switzerland; ' ${ }^{2}$ National Health and Environmental Effects Research Laboratory, US EPA, Research Triangle Park, NC, United States of America

\subsection{6/oemed-2013-101717.387}

Objectives Highway maintenance workers are exposed to elevated particle and noise levels during their work. Exposure to particles as well as to noise has been linked to cardiovascular diseases. Thus, this worker population may be at higher cardiovascular risk. With our study we aimed to provide a better understanding of the workers' exposure and to assess associated short-term health effects.

Methods We monitored the particle and noise exposure of 18 road maintenance workers during five repeated, non-consecutive work shifts and measured health endpoints including blood pressure, selected blood markers for inflammation and thrombosis, continuous ECG as well as lung function and exhaled nitric oxide (FeNO).

Results The workers' exposure to particles and noise was variable between work shifts and depended on work activities. Fine particle $\left(\mathrm{PM}_{2.5}\right)$ mass concentrations over full work shifts ranged from $20.3 \mu \mathrm{g} / \mathrm{m}^{3}$ to $321 \mu \mathrm{g} / \mathrm{m}^{3}$. Particle number concentrations were between $1.6 \mathrm{E} 4$ particles $/ \mathrm{cm}^{3}$ and $4.1 \mathrm{E} 5$ particles $/ \mathrm{cm}^{3}$. Averaged noise levels were frequently above $85 \mathrm{~dB}[\mathrm{~A}]$, ranging from $73.3 \mathrm{~dB}[\mathrm{~A}]$ to $99.6 \mathrm{~dB}[\mathrm{~A}]$. The 17 subjects included for health assessments had an average age of 45.2 years (SD 7.6) and a BMI of $26.6 \mathrm{~kg} / \mathrm{m}^{2}$ (2.6). Average systolic/diastolic blood 